\title{
Elucidating the Molecular interaction of Zebrafish (Danio rerio) Peptidoglycan recognition protein 2 with Diaminopimelic acid and Lysine type Peptidoglycans using in-silico Approaches
}

Rout, Ajaya Kumar; Paramanik, Sunanda; Dehury, Budheswar; Acharya, Varsha; Swain, Himanshu Sekhar; Pradhan, Sukanta Kumar; Behera, Bhaskar; Pati, Soumen Kumar; Behera, Bijay Kumar; Das, Basanta Kumar

Published in:

Journal of Biomolecular Structure and Dynamics

Link to article, DOI:

10.1080/07391102.2019.1666742

Publication date:

2020

Document Version

Peer reviewed version

Link back to DTU Orbit

Citation (APA):

Rout, A. K., Paramanik, S., Dehury, B., Acharya, V., Swain, H. S., Pradhan, S. K., Behera, B., Pati, S. K., Behera, B. K., \& Das, B. K. (2020). Elucidating the Molecular interaction of Zebrafish (Danio rerio) Peptidoglycan recognition protein 2 with Diaminopimelic acid and Lysine type Peptidoglycans using in-silico Approaches. Journal of Biomolecular Structure and Dynamics, 38(12), 3687-3699. https://doi.org/10.1080/07391102.2019.1666742

\section{General rights}

Copyright and moral rights for the publications made accessible in the public portal are retained by the authors and/or other copyright owners and it is a condition of accessing publications that users recognise and abide by the legal requirements associated with these rights.

- Users may download and print one copy of any publication from the public portal for the purpose of private study or research.

- You may not further distribute the material or use it for any profit-making activity or commercial gain

- You may freely distribute the URL identifying the publication in the public portal 


\section{Elucidating the Molecular interaction of Zebrafish (Danio rerio) Peptidoglycan recognition protein 2 with Diaminopimelic acid and Lysine type Peptidoglycans using in-silico Approaches}

Ajaya Kumar Rout, Sunanda Paramanik, Budheswar Dehury, Varsha Acharya, Himanshu Sekhar Swain, Sukanta Kumar Pradhan, Bhaskar Behera, Soumen Kumar Pati, Bijay Kumar Behera \& Basanta Kumar Das

To cite this article: Ajaya Kumar Rout, Sunanda Paramanik, Budheswar Dehury, Varsha Acharya, Himanshu Sekhar Swain, Sukanta Kumar Pradhan, Bhaskar Behera, Soumen Kumar Pati, Bijay Kumar Behera \& Basanta Kumar Das (2019): Elucidating the Molecular interaction of Zebrafish (Danio rerio) Peptidoglycan recognition protein 2 with Diaminopimelic acid and Lysine type Peptidoglycans using in-silico Approaches, Journal of Biomolecular Structure and Dynamics, DOI: 10.1080/07391102.2019.1666742

To link to this article: https://doi.org/10.1080/07391102.2019.1666742

$+\quad$ View supplementary material $\Longleftarrow \pi$

Submit your article to this journal ¿
Accepted author version posted online: 13 Sep 2019.

山 Article views: 13 


\title{
Elucidating the Molecular interaction of Zebrafish (Danio rerio) Peptidoglycan recognition protein 2
} with Diaminopimelic acid and Lysine type Peptidoglycans using in-silico Approaches

Ajaya Kumar Rout ${ }^{1}$, Sunanda Paramanik ${ }^{2}$, Budheswar Dehury ${ }^{3}$, Varsha Acharya ${ }^{1}$, Himanshu Sekhar Swain $^{1}$, Sukanta Kumar Pradhan ${ }^{2}$, Bhaskar Behera ${ }^{4}$, Soumen Kumar Pati ${ }^{5}$, Bijay Kumar Behera ${ }^{1}$, Basanta Kumar $\operatorname{Das}^{1 *}$

${ }^{1}$ Biotechnology Laboratory, ICAR-Central Inland Fisheries Research Institute, Barrackpore, Kolkata, 700120, West Bengal, India

${ }^{2}$ Department of Bioinformatics, Orissa University of Agriculture and Technology, Bhubaneswar, 751003, Odisha, India

${ }^{3}$ Department of Chemistry, Technical University of Denmark, DK-2800 Kongens Lyngby, Denmark

${ }^{4}$ Department of Biosciences and Biotechnology, Fakir Mohan University, Vyasa Vihar, Balasore-756020, Odisha, India

${ }^{5}$ Department of Bioinformatics, Maulana Abul Kalam Azad University of Technology, Haringhata, Nadia741249, West Bengal, India

*Corresponding Authors: Basanta Kumar Das, Email: basantakumard@gmail.com, Tel: +91-033$25921190(\mathrm{O})$, Fax: $+91-033-25920388$

\begin{abstract}
Peptidoglycan recognition proteins (PGRPs) are the important members of pattern recognition receptors (PRRs) family and represents major constituent of innate immunity. Although PGRPs are structurally conserved through evolution; their involvement in innate immunity are different in vertebrates and invertebrates. They are highly specific for the recognition and in some cases, hydrolyze bacterial
\end{abstract}


peptidoglycans (PGNs). Zebrafish PGRPs (zPGRPs) have both peptidoglycans lytic amidase activity and broad-spectrum bactericidal activity, but far less is known about how these receptors recognize these microbial ligands. Such studies are hindered due to lack of structural and functional configuration of zPGRP. Therefore to fill the research gap, we inferred the three-dimensional architecture of the zPGRP2 through theoretical modeling and investigated the conformational and dynamic properties through molecular dynamics simulations. Docking information of microbial ligands i.e., muramyl pentapeptideDAP (MPP-Dap), muramyl pentapeptide-LYS (MPP-Lys), muramyl tripeptide-DAP (MTP-Dap), muramyl tripeptide-Lys (MTP-Lys), muramyl tetrapeptide-DAP (MTr-Dap), muramyl tetrapeptide-LYS (MTr-Lys) and tracheal cytotoxin (TCT) in Autodock Vina revealed $\beta 1, \alpha 2, \alpha 4, \beta 4$, and loops connecting $\beta 1-\alpha 2$, $\alpha 2-\beta 2, \beta 3-\beta 4$, and $\alpha 4-\alpha 5$ as the key interacting domains participate in ligand recognition. Several conserved amino acids i.e., His31, His32, Ala34, Ile35, Pro36, Lys38, Asp60, Trp61, Trp63, Ala89, His90, Asp106, His143, and Arg144 are predicted to essential for binding and provides stability to these zPGRPPGN complexes. Our study provides basic molecular information for further research on the immune mechanisms of PGRP's in Zebrafish. The plasticity of the zPGRP's binding site revealed by these microbial ligands suggests an intrinsic capacity of the innate immune system to rapidly evolve specificities to meet new microbial challenges in the future.

Keywords: Innate immunity, Zebrafish, PGRP 2, Molecular dynamics Simulation, Principal Component Analysis

\section{List of Abbreviations}

BLAST Basic Local Alignment Search Tool

CDD Conserved Domain Database

DAP Diaminopimelic acid

DOPE Discrete Optimized Protein Energy

Lys Lysine 
MPP Muramyl Pentapeptide

MTP Muramyl Tripeptide

MTrP Muramyl Tetrapeptide

NCBI National Centre for Biotechnology Information

NMR Nuclear Magnetic Resonance Spectroscopy

PAMPs Pathogen Associated Molecular Patterns

PDB Protein Data Bank

PGN Peptidoglycan

PGRP Peptidoglycan Recognition Protein

PRRs Pattern Recognition Receptors

RMSD Root Mean Square Deviation

RMSdis Root Mean Square Distance

RMSF Root Mean Square Fluctuation

SOPMA $\quad$ : $\quad$ Self Optimized Prediction Method with Alignments

TCT Tracheal Cytotoxin

TLR5 Toll-Like Receptor 5

VMD Visual molecular dynamics

\section{Introduction}

Innate immunity, the first line of defence against pathogens have evolved and remained an essential component of defence mechanisms in all vertebrates and invertebrates (Hoffmann \& Reichhart, 2002). Innate immunity can discriminate between pathogen and self and start a rapid defensive response through pattern recognition receptors (PRRs) like Nod-like receptors, peptidoglycan recognition proteins (PGRPs), CD14, Toll-like receptors (TLRs), mannose-binding lectin, RegIII $\gamma$ C-type lectin and lysozyme (Royet \& 
Dziarski, 2007) which detect pathogens by the recognition of pathogen-associated including polysaccharides, bacterial DNA and other molecules not normally found on the surface of multicellular organisms, peptidoglycan (PGN), molecular patterns (PAMPs), lipopolysaccharide (LPS), double-stranded viral RNA, (Medzhitov \& Janeway, 2002; Medzhitov, 2007). Along with these PRRs, the first PGRP was discovered from the hemolymph of the silkworm (Bombyx mori) by Ashida and coworkers in 1996. They purified a $19 \mathrm{kDa}$ protein current in the haemolymph of a silkworm (Bombyx mori) that bound activated the prophenoloxidase cascade and peptidoglycan, an antimicrobial defence mechanism in insects (Yoshida et al., 1996). The peptidoglycan recognition protein (PGRP) family is a class of PRRs that are structurally conserved through evolution in vertebrate and invertebrates those binds, and sometimes cleaves, peptidoglycan (PGN), a key component of the bacterial cell wall (Guan \& Mariuzza, 2007). A sum of 13 and 4 PGRP family members have been identified in Drosophila and humans, respectively (Kang et al., 1998; Werner et al., 2000; Liu et al., 2001). PGNs are N-acetylmuramic acid (MurNAc) in $\beta$ (1->4) linkage, cross-linked by short peptide stems and polymers of alternating N-acetylglucosamine (GlcNAc) (Heijenoort, 2001; Doyle \& Dziarski, 2002). The glycan chains demonstrate a little variation among different bacterial species. The third positioned amino acid in these cross-linking peptides differ in Gramnegative bacteria and Gram-positive bacteria: the former is L-lysine (LYS) and the later is mesodiaminopimelic acid (m-DAP) (Dziarski, 2004). Although PGN binding ability plays an essential role in the function of PGRPs, a few PGRPs show selective binding sides towards DAP and LYS type PGNs.

The invertebrate PGRPs like in Drosophila play a vital role in identify PGNs and activating two distinct signalling pathways Toll which is mostly triggered by Lys-type PGNs from Gram-positive bacteria or the immune deficient (IMD) which is primarily activated by Dap-type PGNs from Gram-negative bacteria to regulate gene expression of antimicrobial peptides (AMPs) (Leone et al., 2008; Guan et al., 2004; McDonald et al., 2005). Mouse PGRPs, the intracellular killing bacteria is present in neutrophil tertiary granules (Liu et al., 2000). Mammals have four Peptidoglycan recognition proteins, among them three of 
which were initially identified as bactericidal proteins and one as a peptidoglycan-hydrolytic enzyme, amidases (Royet \& Dziarski, 2007).

Zebrafish and other teleosts fishes have well developed that is similar to the mammalian immune system, unlike invertebrates which includes both innate and adaptive immunity (Yoder et al., 2002; Lam et al., 2004; Trede et al., 2004). Zebrafish is an important model organism for the role of innate immunity and requirement PGRP molecule in defense and survival against bacterial infections in lower vertebrates (Yoder 2002; Lam et al., 2004; Trede et al., 2004; Trede et al., 2001; DeVries et al., 2006). While some functions of zPGRP have been considered, several significant aspects of the interactions among PGRPs and ligands are still indistinguishable. Therefore, further studies verifying these roles of PGRPs are needed. The three-dimensional (3D) model of zebrafish PGRP2 was constructed by in-silico modeling technique. The molecular interaction between zPGRP2 and PGNs were explored using molecular docking, molecular dynamics (MD) simulations, and molecular mechanics/Poisson Boltzmann surface area (MM/PBSA) methods. Our study highlights the crucial intermolecular interactions among PGNs (Lys-PGN and DapPGN) with zPGRP2 and provides novel insights into the PGRP2-PGN ligand mediated interaction in model organism Zebrafish.

\section{Materials and methods}

\section{Data acquisition and identification of functional domains}

The amino acid sequence of ZPGRP2 was retrieved from the NCBI protein database with Accession No: NP_001038631. The Functional domain architecture of zPGRP2 was analyzed by using SMART (Letunic et al., 2011), CDD (Marchler-Bauer et al., 2010) followed by Pfam (Finn et al., 2013). ProtParam (Boeckmann et al., 2003) was aid in depicting various physic-chemical properties of zPGRP2. To detect repeats in protein sequences an automatic algorithm, RADAR (Heger \& Holm, 2000) was used. RADAR is sufficiently fast to scan databases while producing an explicit multiple alignment of each repeat type found.

\section{D structure prediction of zPGRP2}


The primary sequence characterization was conducted using ExPASy-ProtParam tool (Gasteiger et al., 2003) to compute physical and chemical parameters of zPGRP2 protein. The presence and location of signal peptide cleavage sites and disulfide bonds in the protein were predicted using SignalP 4.1 and DiANNA 1.1 (Ferre \& Clote, 2006) web server respectively. Prediction of the secondary structure of protein was carried out using PSIPRED (Buchan et al., 2013). To infer the structure and function of zPGRP2, the PGRP domain region was considered for model building. Suitable templates for 3D model building of zPGRP2 were performed using DELTA-BLAST search against the Protein Data Bank (PDB) database. The top hit identified from BLAST search was selected as best template i.e., crystal structure (PDB ID: 1SXR_A) of the Drosophila Peptidoglycan Recognition Protein for target-template alignment and 3D model building. MODELLER version9.19 (Webb \& Sali, 2014) assisted to build 100 rough models of which the model with lowest discrete optimized protein energy (DOPE) score was finally for further structural modification.

\section{Model validation}

To obtain a realistic 3D structure of zPGRP2, the protein model obtained from Modeller was refined using Galaxy Refine (Heo et al., 2013) web server. The evaluated model of PGRP2 Protein quality was then assessed based on several online validation servers, i.e. WHATIF for side chain optimization (Chinea et al., 1995). Overall quality of the refined model was evaluated to the stereo-chemical geometry and energy. PROCHECK (Laskowski et al., 1993) was used to evaluate the geometry of the 3D models; ERRAT (Colovos \& Yeates, 1993) was used for valuing accuracy of the non-bonded atoms. ProSA-web server was used to evaluate the energy potential of generated 3D model (Wiederstein \& Sippl, 2007). ProQ (Wallner \& Elofsson, 2003) checked the quality of the predicted models. Bond angle and length analyses were performed by using MolProbity web server (Chen et al., 2010).

\section{Molecular Dynamic Simulation}

To recognize the dynamic behavior of zPGRP2 by MD simulation was performed for both protein and ligand using AMBER99SB force field with GROMACS 5.1 package (Abraham et al., 2015). The 
procedure and parameters of molecular dynamics study were adopted from our previous literature (Rout et al. 2018; Rout et al. 2019a; Maharana et al., 2018; Dehury et al., 2013; Dehury et al., 2015). Both the systems were solvated using simple point charge extended (SPC/E) water model in cubic boxes with minimum distance of $10 \AA$ from protein surface to box edge. A physiological ionic strength $(0.15 \mathrm{M}$ of salt $\mathrm{NaCl}$ ) of counter ions was added to neutralize in each system. The average structures were extracted the stable portion of the MD trajectory. Stability of the protein models of zPGRP2 during Molecular Dynamics simulations was analyzed by calculating the root mean square deviation (RMSD), root mean square fluctuation (RMSF), and radius of gyration (Rg). The 3D Structure visualization was carried out by using PyMOL and VMD (Humphrey et al., 1996).

\section{Ligand optimization and Molecular docking}

The ligand structures of muramyl pentapeptide (MPP), muramyl tetrapeptide (MTrP), muramyl tripeptide (MTP), tracheal cytotoxin (TCT) were achieved from the PDB database with PDB IDs: 2APH, 4KNL 1TWQ and 2F2L correspondingly. The lysine residue at $3^{\text {rd }}$ position was carefully replaced with Dap followed by ligand optimization and energy minimization in PRODRG2 server (Schüttelkopf \& Van Aalten, 2004). The final structures of MPP-Dap, MPP-Lys, MTP-Dap, MTP-Lys, MTr-Dap, MTr-Lys and PGRP2-TCT were cross-checked with the synthetic PGN compounds. The 3D coordinates of these ligands along with TCT were considered for docking simulation studies with zPGRP2. The following procedure was implemented from literature study (Sahoo et al., 2014; Qi et al., 2017). In this study we used, AutoDock Vina (henceforth referred to as ADVINA), a freely available program for academic and commercial applications. ADVINA provides some degree of protein flexibility by allowing predefined side-chain residues to be flexible during docking. ADVINA 1.0.2 (Trott \& Olson, 2010) was used to perform docking using all the flexible and rigid zPGRP2 conformations. A docking grid with a size of $60 \times 60 \times 60$ was used. For each PDB structure, the center co-ordinate was obtained from the central atom of the co-crystallized inhibitor. The grid size was selected in order to encompass the co-crystallized inhibitors

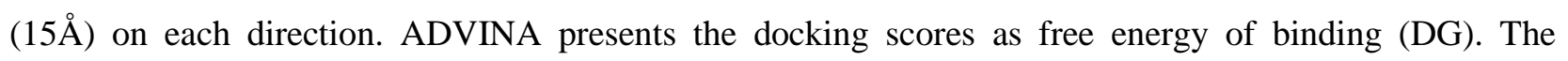


resultant protein-ligands interactions were plotted with pymol and ligplot (2D plot) to show their H-bond, pi-bond and electrostatic interactions.

\section{Principal component analysis}

Principal component analysis (PCA) is multivariate statistical methods which separate the collective motions and conformational changes in protein and protein-Ligand complex using GROMCAS tools (Dikhit et al., 2017; Dikhit et al., 2018). To understand the collective motion of zPGRP2 with MPP-Dap, MPP-Lys, MTP-Dap, MTP-Lys, MTr-Dap, MTr-Lys and PGRP2-TCT conformations, PCA was performed by using g_covera and g_aneig tools (Maharana et al., 2014a; Maharana et al., 2014b; Maharana 2015; Rout et al., 2019b).

\section{Binding free energy calculation}

The binding energy calculation of various MPP-Dap, MPP-Lys, MTP-Dap, MTP-Lys, MTr-Dap, MTr-Lys and PGRP2-TCT complex systems by using g_mmpbsa v5.12 script was carried out using GROMACS. The (MM/PBSA) method use to compute the binding free energy calculation.

The MM/PBSA ( $\triangle \mathrm{G}_{\mathrm{bind}}$ ) was calculated the following methods:

$\Delta \mathrm{G}_{\text {bind }}=\mathrm{G}_{\text {complex }}-\mathrm{G}_{\text {protein }}-\mathrm{G}_{\text {ligand }}=\Delta_{\mathrm{EMM}}+\Delta_{\text {Gsol }}-\mathrm{T} \Delta \mathrm{S}(1)$

$\Delta \mathrm{E}_{\mathrm{MM}}=\Delta \mathrm{E}_{\text {bonded }}+\Delta \mathrm{E}_{\text {nonbonded }}=\Delta \mathrm{E}_{\text {bonded }}+\left(\Delta \mathrm{E}_{\mathrm{vdw}}+\Delta \mathrm{E}_{\text {ele }}\right)(2)$

$\Delta \mathrm{G}_{\mathrm{sol}}=\Delta \mathrm{G}_{\mathrm{polar}}+\Delta \mathrm{G}_{\mathrm{nonpolar}}(3)$

The binding energy calculations were analyzed from GROMACS, the parameter for binding free energy calculation was adopted from previous published literature (Dehury et al., 2017; Das et al., 2019; Roy et al., 2019). 500 snapshots were extracted from the last $10 \mathrm{~ns}$ equlibrated trajectory of 50ns MD simulations.

\section{Results}

\section{In silico characterization of zPGRP2}

Danio rerio peptidoglycan recognition protein (zPGRP2) sequence (458 amino acids) was retrieved from NCBI protein database (NP_001038631). The domain prediction of PGRP2 by SMART and Pfam revealed two putative domains that are overlapping with each other viz., PGRP (285-431) and Ami-2 (enzyme 
domain (297-438). The amino acid sequences of Danio rerio PGRP2 with other organism were used to draw phylogenetic relationships among them by using MEGA version 6.0 (Kumar et al., 2016). The zPGRP2 sequence was compared with other nine different organisms using CLUSTAL W and a molecular phylogenetic tree was build based on pairwise alignments with iteration of 1000 bootstrap value. The evolutionary history was inferred using the NJ Method. The optimal tree with the sum of branch length = 3.79 is shown in Figure S1. The percentage of replicate trees in which the associated taxa clustered together in the bootstrap test (1000 replicates) is shown next to the branches. Based on sequence phylogenetic analysis of zPGRP2 with it closes homologs, Ctenopharyngodon idella is closely related to

\section{Danio rerio (Figure S1).}

\section{Comparative modeling and Structure Quality Assessment}

Template search for zPGRP2 in PDB using BLASTp showed the crystal structure of Chain A, Drosophila peptidoglycan recognition protein (PGRP-SA) (PDB ID: 1SXR_A) shared the highest homology with an identity of 37\% and similarity 59\% (Figure S2). The secondary structure analysis by PSIPRED server (Figure S3) illustrate that coils/turns dominate over helices and strands along with the secondary structure elements (Figure S4). Considering the above homology, a target template alignment was drawn and thereafter 50 models built, among which the best model was identified by analyzing the lowest DOPE score. The model structure was further refined and energy minimized by Galaxy Refine and presented before different validation servers to optimize its potential.

The simulated zPGRP2 model comprised of $4 \alpha$-helices and $5 \beta$-strands (one parallel and one anti-parallel). The inner core of model is formed of $\beta$-sheets while the outer surface surrounded by helices (Figure 1A). The accuracy of dihedral angles $(\Phi / \Psi)$ of zPGRP2 model was measured using Ramachandran plot in Procheck programme (Figure 1B). The refined model showed good percent of residues in most favored regions (112 amino acids, 93.3\%), additional allowed regions (6 amino acids, 5.0\%) and disallowed regions (2 amino acids. 1.7\%). The statistics of non-bonded interactions between different atom types calculated by ERRAT also revealed a good validation score (95.652), and suggesting the reliability of the 
initial model. Energy profile of the proposed model and the z-score value was obtained using ProSA program which calculates the interaction energy per residue using a distance-based pair potential. ProSA revealed a z-score value of the model which lies between -5.0 to -9.0 (negative value imply model accuracy) as depicted in Figure 1C. ProQ analysis indicated that the quality of zPGRP2 was extremely good. The details of the Protein validation information have been summarized in Table 1.

\section{Molecular docking of protein-ligand complexes}

The zPGRP2 consisted of two potential N-glycosylation sites, among these the N79 and N127 were situated on the loop surrounded by the L-shaped cleft. Earlier studies in different PGRP receptors highlighted this region as the potential binding region of PGN compounds. The large molecular size of muramyl-peptides with more than $\sim 25$ rotatable bonds may not be very accurate because of algorithm limitations. The docking results in AutoDock Vina generated good binding energy for all constructed PGN compounds, and the binding energy obtained in both programs has been listed in Table 2.

The zPGRP2-MPP-Dap showed that, some crucial residue i.e. Ala34, Ile35, Lys38, Trp61, Ala89 (as shown in Figure 2A), while His32, Ala89, His90, Lys92, and Arg144 were found zPGRP2-MPP-Lys interaction was elucidated in Figure 2B. In contrast, His31, Asp60 Trp61, Trp63, and His143, were also found to be actively engaged in zPGRP2-MTP-Dap type interactions (See Figure 2C).

The binding sites of C-terminal peptide stems interaction were varied significantly with different orientations. The ligand binding pockets and corresponding the atomic interaction plots between zPGRP2MTP-Lys ligands and residue His31, Ala34, Trp61, Ala89, His90, Lys92, His143, and Arg144 were shown in Figure 3A and His31, Ile35, Lys38, Trp61, Pro63, Trp63, His90, Ala89, His143 in Figure 3B respectively. The MTr-Lys rendered an approximately same binding orientation for all muramyl peptides and actively bonded with the His31, His32, Asp60, Trp61, Ala89, His143 and Arg144 residues of zPGRP2 (See Figure 4A). For zPGRP2-TCT, docking interactions of His31, Ala34, Ile35, Trp61, His143, and Arg144 are illustrated in Figure 4B.

\section{MD simulation}


To ensure the stability of modeled zPGRP2, MD simulation of the model and the complexes were performed over a simulation time period of 50ns. All systems are attained equilibrium during 50ns MD simulation as revealed from the potential energy (data not shown). The backbone RMSD of the each system plotted as a function of time revealed the stability of the system. The RMSD analysis of the backbone atoms showed an initial upraise till $\sim 30 \mathrm{~ns}$, and thereafter achieved a steady plateau with an average RMSD value of $\sim 3.53 \AA$ (Figure 5A). We observed differential variation in RMSD pattern for different PGPN ligands as compared to apo system, which may be due to slight change in the orientation of ligand within the binding pocket of each complex. Like RMSD, the PGRP2-MTP-Dap and PGRP2-MTPLys systems showed slightly differential pattern in Rg as compared to the other systems. The radius of gyration $(\mathrm{Rg})$ analysis revealed a gyration radii of $\sim 14.30 \AA$ indicates the compactness of each complex system (Figure 5B). To understand the residual fluctuation of the model, the root mean square fluctuations (RMSF) of the $\mathrm{C} \alpha$ atoms were calculated RMSF analysis with respect to simulation time (as displayed in Figure 5C). The residual fluctuation analysis from RMSF analysis clearly portrayed that all ZPGRP2MPP-Dap, MTP-Dap, and MTR-Dap complex systems exhibits high degree of fluctuation as compared to the other systems. The high degree of flexibility was observed in the loop and ligand binding regions in most of systems signifies their participation in PGN ligand recognition.

As compared to $\mathrm{N}$-terminal region, the $\mathrm{C}$-terminal loop regions in zPGRP2 comprised of 25 residues exhibited the maximum fluctuation $(>4 \AA)$. Intermolecular contact analysis of ligands with zPGRP2 has been summarized in Table S1. Using g_hbond utility toolkit, the dynamics of hydrogen bond (H-bond) over the 50 ns was computed and presented in Figure 6. The MPP-Dap system possess a stable trend in H-bonding pattern with average of $\sim 5.00$ bonds (Figure 6A); MPP-Lys was ( 5.2 ) details see Figure 6B; MTP-Dap had H-bond of 7.20 as shown in Figure 6C; MTP-Lys was coupled with number of H-bonds ( 8.2) Figure 6D; MTr-Dap of H-bond ( 6.8) in Figure 6E; MTr-Lys of h-bond ( 4.8) in Figure 6F; TCT maintain the upper number of H-bonds ( $~ 8.00)$ in Figure 6G Respectively.

\section{Essential Dynamics}


In the present study, the first two principal components i.e., PC1 and PC2 which holds the maximum global motion of each system were analyzed in detail (Figure 7). The resultant MD trajectories were used for PCA to determine the projection of the first two eigenvectors (EVs). Movements of C $\alpha$ atoms were found to be all system as evidenced from porcupine plot (Figure 8). The trace value of covariance matrix of zPGRP2 (apo) was $9.99 \mathrm{~nm}^{2}$. MPP-DAP covariance matrix was $11.43 \mathrm{~nm}^{2}$ (Figure 8B); MPP-LYS covariance matrix was $11.69 \mathrm{~nm}^{2}$ (Figure 8C); TCT covariance matrix was $9.96 \mathrm{~nm}^{2}$ (Figure 8D); MTPDAP covariance matrix was $15.23 \mathrm{~nm}^{2}$ (Figure 8E); MTP-LYS covariance matrix was $14.25 \mathrm{~nm}^{2}$ (Figure 8F); MTR-DAP covariance matrix was $15.50 \mathrm{~nm}^{2}$ (Figure 8G); and MTR-LYS covariance matrix was $13.13 \mathrm{~nm}^{2}$ (Figure 8H) respectively. As compared to the apo system, the holo systems (mostly the MTPDAP, MTP-LYS and MTR-DAP) occupied more conformational space implies that these systems are highly flexible in nature.

\section{MM/PBSA Binding Free Energy}

We computed the free energy of each system using MM/PBSA approach, which is are arguably one of the important and popular method for binding free energy prediction due to its accuracy than scoring functions implemented in molecular docking tools. Using 500 snapshots from last $10 \mathrm{~ns}$ MD trajectory of each system, we computed binding free energies of zPGRP2-ligand complexes. Table 3 summarizes the computed energy terms for each complex i.e., electrostatic, van der Waals, polar solvation, and SASA energies contributing to the overall free energy of the system. In two systems (as shown in Table 3), we computed the binding free energy to be positive. In all PGRP2-PGN complex systems, van der Waals and SASA were negative and polar salvation terms were positive. This signifies that van der Waals and SASA favored the binding, while the polar salvation terms opposed the binding. In addition, we found that electrostatic energy also contributes to the overall binding free energy of each system. It is noteworthy to note that the computed binding free energy cannot be compared with experimental binding energy, as MM/PBSA approach employed in this study ignores the entropic contribution while computing the binding free energy. 


\section{Discussion}

The crucial structural information about the PGRPs in case of zebrafish encounter to the microorganism having a great attention in recent studies (Guan et al., 2004). The zzPGRP2 components functioning in antimicrobial defenses and have accomplish their functions through different protein based mechanisms. Therefore, it is essential to sketch out the molecular interaction between the zPGRP2 receptor with DAP and LYS-type peptidoglycans for survival of zebrafish developing embryo from bacterial infection (Li et al., 2007). Details in-sillico research design explained the real structural validation and molecular dynamics of Zebrafish (Danio rerio) zPGRP2 receptor protein targeted the respective peptidoglycans (DAP and LYS-type) components.

The molecular weight of zPGRP2 (147aa long) was 53212.8 KDa. Physico-chemical property analysis showed that zPGRP2 is basic in nature ( $\mathrm{pI}>7.0$ ). The computed isoelectric point (8.55) will be useful for developing buffer system for purification by isoelectric focusing method. The aliphatic side chains are regarded as a positive factor for the increase of thermal stability of proteins (Ikai, 1980). Aliphatic index of PGRP2 is 76.87. High aliphatic index of PGRP2 indicates that of Danio rerio PGRP2 protein may be stable for a wide range of temperature. The instability index less than 40 indicates stable nature of the protein in test tube. Hydropathy analysis of zPGRP2 revealed that the protein is hydrophilic in nature. Analysis of the sequence with SignalP 4.1 tool did not predict any signal peptide in zPGRP2 protein. The $\mathrm{N}$-glycosylation sites prediction using NetNGlyc 1.0 server proposed six glycosylation sites, out of which two were highly potential (N79 and N203), two moderate (N51 and N203), and two (N62and N208) predicted to be with low potential. These potential glycosylation sites suggested their role in PGN ligand recognition and bactericidal activity (Guan 2004; Reiser et al., 2004).

Disulfide bond prediction at DiANNA showed the presence of one potential bond between C40-C46 residues. The SOPMA showed that the protein contains $14.97 \%$ alpha helix, with random coil is $42.86 \%$ and beta turn is $14.97 \%$. The extended strand is $27.21 \%$. The abundance of coiled region indicates higher conservation. 
The ligand binding conserved domain (CD) of zPGRP2 belongs to super family c102712 and homologous to the T3 lysozyme of Bacteriophage. Applying the PRALINE server for multiple sequence alignment of different receptor proteins (PGRP2, PGRP5, PGRP6) of zebrafish advocated about the homology between the sequences and their effectual potentiality (Figure S5). The high conserved region within those domains depicts their strong evolutionary relationships and the hydrophobicity about the PGRP2, which is more hydrophobic nature than the other two PGRPs in zebrafish. The consistencies of the amino acid conservation percentage in a column represents in colour gradient to the accurate prediction of hydrophobicity nature.

The sequence alignment zPGRP2 with the (PGRP)-SA (1SXR) of Drosophila revealed that the N-terminal end is the less conserved as compared to C-terminal end, while the disulphide bridge forming cysteine residues (Figure S2) were found to be strongly conserved (Reiser et al., 2004). Like other PGRPs from human and Drosophila, the zebrafish PGRP2 model was comprised of multiple four $\alpha$-helices and five $\beta$ sheet structures (Guan et al., 2004, Leone et al., 2008).

Molecular docking calculations were performed between PGN ligands and modelled zPGRP2. Docking results displayed presented in Table 2 indicated most of ligands had similar docking scores where the binding poses of Lys- and Dap-type ligands resides in the extended conformation at the deep end of the binding cleft with variable of intermolecular contacts dominated by H-bonds. The mode of binding of these PGN ligands is well aligned with previous in silico study in buffalo PGRP1 (Sahoo et al., 2014). For the zPGRP2-TCT complex, in addition to H-bonds, electrostatic interaction was found to be crucial for molecular interaction. All these PGN ligands formed more than $6 \mathrm{H}$-bonds where few residues including His31, Trp61, Ala89, His143, and Arg144 repeatedly participated in H-bond interaction. This indicates that these residues might play important roles in the zPGRP2 selective binding to Lys-PGN and Dap-PGN. Both type ligands shared approximately same interacting sites for the acetylmuramic acid atoms in zPGRP2; however, varied at the peptide stem and $3^{\text {rd }}$ place comprising L-Lys or Dap binding sites. The key binding residues predicted were well compatible with the previous in-silico (Sahoo et al., 2014) and 
experimental studies Drosophila (Reiser et al., 2004). The His31 and Arg144 that were actively engaged in acetylmuramic acid binding may be highly responsible for the PGN-fragment orientation as revealed from previous studies (Mellroth et al., 2003; Guan et al., 2005) and upon mutation may affect the stability of the complex. This indicated, in Zebrafish PGRP2, the His31 and Arg144 residues may be highly essential to mediate the bactericidal activity and PGRP2-sense innate immune signaling. The observation from docking simulation studies are in agreement fish PGRP6 which can also bind to Lys-type and Dap-type PGNs (Li et al., 2014).

The RMSDs of backbone atoms and radius of gyration of over the $50 \mathrm{~ns}$ simulation time for zPGRP2-PGN complex systems displayed variable trend, where MTP-Dap and MTP-Lys displayed larger deviation than other PGNs. The RMSD of the other four PGN complexes showed relative plateau with a least backbone deviations as compared to the MTP-Dap and MTP-Lys systems. All the above indicated that all these complexes were well equilibrated during MD simulations. Inter-molecular contact analysis from snapshots from MD trajectories revealed that van der Waals interaction contributes more to the overall free binding energy in most of the complexes. Similar observations were reported in human buffalo and human PGRPs (Sahoo et al., 2014; Lim et al., 2006). Thus, we assumed that zebrafish PGRPs have similar amino acid residues like those of fish (Wang et al., 2018), human (Lim et al., 2006), buffalo (Sahoo et al., 2014) and drosophila (Leone et al., 2008) PGRPs which dictate the selective differential binding of Lys- and DapPGNs.

\section{Conclusion}

Several studies on PGRP-sense innate signalling has been reported in human and Drosophila. The binding site of Gram positive and negative bacteria cell-wall PGN fragments in PGRP is very imperative to understand these mechanisms. In this study, we reported the key binding domains and amino acid residues that recognize the PGN structures of both Gram positive and negative bacteria. Results showed the Daptype PGN has a greater binding affinity to PGRP2 receptor than Lys-type in Zebrafish. The conserved amino acid residues sensing the acetylmuramic acid and D-Ala in both Dap- and Lys type PGN-fragments 
warrants in vivo investigation to understand the hydrolyzing and bactericidal activity of PGRP2 in Zebrafish and other fish species. The Dap- and Lys-type PGN key binding domains suggests the need of further investigation for the therapeutic application of the PGRP2 related disorders and innate immune signalling in teleosts and other higher eukaryotes.

\section{Conflict of Interest}

The authors have declared that no competing interests exist.

\section{Acknowledgement}

The authors thank Mr. Jitendra Maharana, Senior Research Fellow, Assam Agricultural University, Jorhat Assam for helping us in generating the figures of our manuscript. We also thank Mr. Asim Kumar Jana, Senior Technical Assistant, ICAR-CIFRI, Barrackpore for technical Support. The First author thanks Mr. Umasankar Ram and Mr. Amarnath Prasad, Support Staff, ICAR-CIFRI, Barrackpore, Kolkata, India.

\section{References}

Abraham, M. J., Murtola, T., Schulz, R., Páll, S., Smith, J. C., Hess, B., \& Lindahl, E. (2015). GROMACS: High performance molecular simulations through multi-level parallelism from laptops to supercomputers. SoftwareX, 1, 19-25. doi.org/10.1016/j.softx. 2015.06.001

Buchan, D. W., Minneci, F., Nugent, T. C., Bryson, K., \& Jones, D. T. (2013). Scalable web services for the PSIPRED Protein Analysis Workbench. Nucleic acids research, 41(W1), W349-W357. doi.org/10.1093/nar/gkt381

Boeckmann, B., Bairoch, A., Apweiler, R., Blatter, M. C., Estreicher, A., Gasteiger, E., ... \& Pilbout, S. (2003). The SWISS-PROT protein knowledgebase and its supplement TrEMBL in 2003. Nucleic acids research, 31(1), 365-370. doi.org/10.1093/nar/gkg095

Chen, V.B., Arendall, W.B., Headd, J.J., Keedy, D.A., Immormino, R.M., Kapral, G.J., Murray, L.W., Richardson, J.S. and Richardson, D.C. (2010). MolProbity: all-atom structure validation for macromolecular crystallography. Acta Crystallographica Section D: Biological Crystallography, 66, 12-21. doi.org/10.1107/S0907444909042073

Colovos, C., \& Yeates, T. O. (1993). Verification of protein structures: patterns of nonbonded atomic interactions. Protein science, 2(9), 1511-1519. doi.org/10.1002/pro. 5560020916 
Chinea, G., Padron, G., Hooft, R. W. W., Sander, C., \& Vriend, G. (1995). The use of position-specific rotamers in model building by homology. Proteins: Structure, Function, and Bioinformatics, 23(3), 415-421. doi.org/10.1093/nar/gkt458

Das, B. K., Roy, P., Rout, A. K., Sahoo, D. R., Panda, S. P., Pattanaik, S., ... \& Mishra, S. S. (2019). Molecular cloning, GTP recognition mechanism and tissue-specific expression profiling of myxovirus resistance (Mx) protein in Labeo rohita (Hamilton) after Poly I: C induction. Scientific reports, 9(1), 3956. doi.org/10.1038/s41598-019-40323-0

Dehury, B., Behera, S. K., \& Mahapatra, N. (2017). Structural dynamics of casein kinase I(CKI) from malarial parasite Plasmodium falciparum (Isolate 3D7): insights from theoretical modelling and molecular simulations. Journal of Molecular Graphics and Modelling, 71, 154-166. doi.org/10.1016/j.jmgm.2016.11.012

Dehury, B., Sahu, M., Sahu, J., Sarma, K., Sen, P., Modi, M.K., Barooah, M. and Choudhury, M.D. (2013). Structural analysis and molecular dynamics simulations of novel $\delta$-endotoxin Cry1Id from Bacillus thuringiensis to pave the way for development of novel fusion proteins against insect pests of crops. Journal of molecular modeling, 19, 5301-5316. doi: 10.1007/s00894-013-2010-X

Dehury, B., Maharana, J., Sahoo, B.R., Sahu, J., Sen, P., Modi, M.K. and Barooah, M. (2015). Molecular recognition of avirulence protein (avrxa5) by eukaryotic transcription factor xa5 of rice (Oryza sativa L.): Insights from molecular dynamics simulations. Journal of Molecular Graphics and Modelling, 57, 49-61. doi.org/10.1016/j.jmgm.2015.01.005

DeVries, M. E., Kelvin, A. A., Xu, L., Ran, L., Robinson, J., \& Kelvin, D. J. (2006). Defining the origins and evolution of the chemokine/chemokine receptor system. The Journal of Immunology, 176(1), 401415. doi.org/10.4049/jimmunol.176.1.401

Dikhit, M. R., Kumar, A., Das, S., Dehury, B., Rout, A. K., Jamal, F., ... \& Bimal, S. (2017). Identification of potential MHC Class-II-restricted epitopes derived from Leishmania donovani antigens by reverse vaccinology and evaluation of their CD4+ T-cell responsiveness against visceral leishmaniasis. Frontiers in immunology, 8, 1763. doi.org/10.3389/fimmu.2017.01763

Dikhit, M. R., Das, S., Mahantesh, V., Kumar, A., Singh, A. K., Dehury, B., ... \& Pandey, K. (2018). The potential HLA Class I-restricted epitopes derived from LeIF and TSA of Leishmania donovani evoke anti-leishmania CD8+ T lymphocyte response. Scientific reports, 8. doi.org/10.1038/s41598-01832040-X

Doyle, R. J., \& Dziarski, R. (2002). The bacterial cell: peptidoglycan. In Molecular medical microbiology (pp. 137-154). Academic Press. doi.org/10.1016/B978-012677530-3/50226-9 
Dziarski, R. (2004). Peptidoglycan recognition proteins (PGRPs). Molecular immunology, 40(12), 877886. doi.org/10.1016/j.molimm.2003.10.011

Ferrè, F., \& Clote, P. (2006). DiANNA 1.1: an extension of the DiANNA web server for ternary cysteine classification. Nucleic acids research, 34(suppl_2), W182-W185. doi.org/10.1093/nar/gk1189

Finn, R. D., Bateman, A., Clements, J., Coggill, P., Eberhardt, R. Y., Eddy, S. R., ... \& Sonnhammer, E. L. (2013). Pfam: the protein families database. Nucleic acids research, 42(D1), D222-D230. doi.org/10.1093/nar/gkt1223

Gasteiger, E., Gattiker, A., Hoogland, C., Ivanyi, I., Appel, R. D., \& Bairoch, A. (2003). ExPASy: the proteomics server for in-depth protein knowledge and analysis. Nucleic acids research, 31(13), 37843788. doi.org/10.1093/nar/gkg563

Guan, R., Wang, Q., Sundberg, E. J., \& Mariuzza, R. A. (2005). Crystal structure of human peptidoglycan recognition protein S (PGRP-S) at $1.70 \AA$ A resolution. Journal of molecular biology, 347(4), 683-691. doi.org /10.1016/j.jmb.2005.01.070

Guan, R., Roychowdhury, A., Ember, B., Kumar, S., Boons, G. J., \& Mariuzza, R. A. (2004). Structural basis for peptidoglycan binding by peptidoglycan recognition proteins. Proceedings of the National Academy of Sciences, 101(49), 17168-17173. doi.org/ 10.1073/pnas.0407856101

Guan, R., \& Mariuzza, R. A. (2007). Peptidoglycan recognition proteins of the innate immune system. Trends in microbiology, 15(3), 127-134. doi.org/10.1016/j.tim.2007. 01.006

Heger, A., \& Holm, L. (2000). Rapid automatic detection and alignment of repeats in protein sequences.

Proteins: Structure, Function, and Bioinformatics, 41(2), 224-237. doi.org/10.1002/1097-0134 (20001101)41:2<224:: AID-PROT70>3.0.CO;2-Z

Heo, L., Park, H., \& Seok, C. (2013). GalaxyRefine: protein structure refinement driven by side-chain repacking. Nucleic acids research, 41(W1), W384-W388. doi.org/10.1093/nar/gkt458

Humphrey, W., Dalke, A., \& Schulten, K. (1996). VMD: visual molecular dynamics. Journal of molecular graphics, 14(1), 33-38. doi.org/10.1016/0263-7855 (96)00018-5

Heijenoort, J. V. (2001). Formation of the glycan chains in the synthesis of bacterial peptidoglycan. Glycobiology, 11(3), 25R-36R. doi.org/10.1093/glycob/11.3.25R

Hoffmann, J. A., \& Reichhart, J. M. (2002). Drosophila innate immunity: an evolutionary perspective. Nature immunology, 3(2), 121. doi.org/10.1038/ni0202-121

Ikai, A. (1980). Thermostability and aliphatic index of globular proteins. The Journal of Biochemistry, 88(6), 1895-1898. doi.org /10.1093/oxfordjournals.jbchem.a133168 
Kang, D., Liu, G., Lundström, A., Gelius, E., \& Steiner, H. (1998). A peptidoglycan recognition protein in innate immunity conserved from insects to humans. Proceedings of the National Academy of Sciences, 95(17), 10078-10082. doi.org/10.1073/pnas. 95. 17. 10078

Kumar, S., Stecher, G. and Tamura, K. (2016). MEGA7: molecular evolutionary genetics analysis version 7.0 for bigger datasets. Molecular biology and evolution, 33, 1870-1874. doi.org/10.1093/molbev/msw054

Laskowski, R. A., MacArthur, M. W., Moss, D. S., \& Thornton, J. M. (1993). PROCHECK: a program to check the stereochemical quality of protein structures. Journal of applied crystallography, 26(2), 283291. doi.org/10.1107/S0021889892009944

Lam, S. H., Chua, H. L., Gong, Z., Lam, T. J., \& Sin, Y. M. (2004). Development and maturation of the immune system in zebrafish, Danio rerio: a gene expression profiling, in situ hybridization and immunological study. Developmental \& Comparative Immunology, 28(1), 9-28. doi.org/ 10.1016/S0145-305X(03)00103-4

Leone, P., Bischoff, V., Kellenberger, C., Hetru, C., Royet, J., \& Roussel, A. (2008). Crystal structure of Drosophila PGRP-SD suggests binding to DAP-type but not lysine-type peptidoglycan. Molecular immunology, 45(9), 2521-2530. doi.org/10.1016/j.molimm. 2008.01.015

Letunic, I., Doerks, T., \& Bork, P. (2011). SMART 7: recent updates to the protein domain annotation resource. Nucleic acids research, 40(D1), D302-D305. doi.org/10.1093/nar/ gkr931

Li, X., Wang, S., Qi, J., Echtenkamp, S. F., Chatterjee, R., Wang, M., ... \& Gupta, D. (2007). Zebrafish peptidoglycan recognition proteins are bactericidal amidases essential for defense against bacterial infections. Immunity, 27(3), 518-529. doi.org/10.1016/j.immuni. 2007.07.020

Li, J. H., Yu, Z. L., Xue, N. N., Zou, P. F., Hu, J. Y., Nie, P., \& Chang, M. X. (2014). Molecular cloning and functional characterization of peptidoglycan recognition protein 6 in grass carp Ctenopharyngodon idella.) Developmental \& Comparative Immunology, 42(2), 244-255. doi.org/10.1016/j.dci.2013.09.014

Lim, J. H., Kim, M. S., Kim, H. E., Yano, T., Oshima, Y., Aggarwal, K., ... \& Oh, B. H. (2006). Structural basis for preferential recognition of diaminopimelic acid-type peptidoglycan by a subset of peptidoglycan recognition proteins. Journal of Biological Chemistry, 281(12), 8286-8295. DOI: 10.1074/jbc.M513030200

Liu, C., Xu, Z., Gupta, D., \& Dziarski, R. (2001). Peptidoglycan recognition proteins a novel family of four human innate immunity pattern recognition molecules. Journal of Biological Chemistry, 276(37), 34686-34694. doi.org/10.1074/jbc.M105566200 
Liu, C., Gelius, E., Liu, G., Steiner, H., \& Dziarski, R. (2000). Mammalian peptidoglycan recognition protein binds peptidoglycan with high affinity, is expressed in neutrophils, and inhibits bacterial growth. Journal of Biological Chemistry, 275(32), 24490-24499. doi.org/10.1074/jbc.M001239200

Maharana J., Panda D., De S. (2018). Deciphering the ATP-binding mechanism (s) in NLRP-NACHT 3D models using structural bioinformatics approaches. PloS one. 13(12), e0209420. doi.org/10.1371/journal.pone.0209420

Maharana, J., Patra, M.C., De, B.C., Sahoo, B.R., Behera, B.K., De, S. \& Pradhan, S.K. (2014a). Structural insights into the MDP binding and CARD-CARD interaction in zebrafish (Danio rerio) NOD2: a molecular dynamics approach. Journal of Molecular Recognition, 27(5), 260-275. doi.org/10.1002/jmr.2357

Maharana, J., Sahoo, B. R., Bej, A., Patra, M. C., Dehury, B., Bhoi, G. K., ... \& Behera, B. K. (2014b). Structural and functional investigation of zebrafish (Danio rerio) NOD1 leucine rich repeat domain and its interaction with iE-DAP. Molecular BioSystems, 10(11), 2942-2953. doi.org/10.1039/C4MB00212A

Maharana, J., Sahoo, B. R., Bej, A., Jena, I., Parida, A., Sahoo, J. R., .. \& Pradhan, S. K. (2015). Structural models of zebrafish (Danio rerio) NOD1 and NOD2 NACHT domains suggest differential ATP binding orientations: insights from computational modeling, docking and molecular dynamics simulations. PloS one, 10(3), e0121415. doi.org/10.1371/ journal.pone.0121415

Marchler-Bauer, A., Lu, S., Anderson, J. B., Chitsaz, F., Derbyshire, M. K., DeWeese-Scott, C., ... \& Gwadz, M. (2010). CDD: a Conserved Domain Database for the functional annotation of proteins. Nucleic acids research, 39(suppl_1), D225-D229. doi.org/10.1093/nar/gkq1189

McDonald, C., Inohara, N., \& Nuñez, G. (2005). Peptidoglycan signaling in innate immunity and inflammatory disease. Journal of Biological Chemistry, 280(21), 20177-20180. doi.org/ 10.1074/jbc.R500001200

Mellroth, P., Karlsson, J., \& Steiner, H. (2003). A scavenger function for a Drosophila Peptidoglycan recognition protein. Journal of Biological Chemistry, 278(9), 7059-7064. doi.org/10.1074/jbc. M208900200

Medzhitov, R., \& Janeway Jr, C. A. (2002). Innate immune recognition. Annu Rev Immunol, 20(2), 197216. doi.org/10.1038/ni0202-121

Medzhitov, R. (2007). Recognition of microorganisms and activation of the immune response. Nature, 449(7164), 819. doi.org/10.1038/nature06246

Qi, Z., Meng, F., Zhang, Q., Wang, Z., Qiao, G., Xu, W., ... \& Chen, C. (2017). Structural insights into ligand binding of PGRP1 splice variants in Chinese giant salamander (Andrias davidianus) from 
molecular dynamics and free energy calculations. Journal of molecular modeling, 23(4), 135. DOI 10.1007/s00894-017-3315-y

Reiser, J. B., Teyton, L., \& Wilson, I. A. (2004). Crystal structure of the Drosophila peptidoglycan recognition protein (PGRP)-SA at $1.56 \AA$ resolution. Journal of molecular biology, 340(4), 909-917. doi.org /10.1016/j.jmb.2004.04.077

Rout, A. K., Dehury, B., Maharana, J., Nayak, C., Baisvar, V. S., Behera, B. K., \& Das, B. K. (2018). Deep insights into the mode of ATP-binding mechanism in Zebrafish cyclin-dependent protein kinase-like 1 (zCDKL1): A molecular dynamics approach. Journal of Molecular Graphics and Modelling, 81, 175183. doi.org/10.1016/j.jmgm.2018.02.002

Rout, A. K., Mishra, J., Dehury, B., Maharana, J., Acharya, V., Karna, S. K., ... \& Das, B. K. (2019). Structural bioinformatics insights into ATP binding mechanism in zebrafish (Danio rerio) cyclindependent kinase-like 5 (zCDKL5) protein. Journal of cellular biochemistry. 120, 94379447.doi.org/10.1002/jcb.28219

Rout, A. K., Udgata, S. R., Dehury, B., Pradhan, S. P., Swain, H. S., Behera, B. K., \& Das, B. K. (2019). Structural bioinformatics insights into the CARD-CARD interaction mediated by the mitochondrial antiviral-signaling protein of black carp. Journal of cellular biochemistry. 1-10. doi.org/10.1002/jcb.28519

Roy, P., Rout, A.K., Maharana, J., Sahoo, D.R., Panda, S.P., Pal, A., Nayak, K.K., Behera, B.K. and Das, B.K., 2019. Molecular characterization, constitutive expression and GTP binding mechanism of Cirrhinus mrigala (Hamilton, 1822) Myxoyirus resistance (Mx) protein. International journal of biological macromolecules, 136 (2019) 1258-1272.

Royet, J., \& Dziarski, R. (2007). Peptidoglycan recognition proteins: pleiotropic sensors and effectors of antimicrobial defences. Nature Reviews Microbiology, 5(4), 264. doi.org/10.1038/nrmicro1620

Sahoo, B. R., Dubey, P. K., Goyal, S., Bhoi, G. K., Lenka, S. K., Maharana, J., ... \& Kataria, R. S. (2014). Exploration of the binding modes of buffalo PGRP1 receptor complexed with meso-diaminopimelic acid and lysine-type peptidoglycans by molecular dynamics simulation and free energy calculation. Chemico-biological interactions, 220, 255-268. doi.org/10.1016/j.cbi.2014.06.028

Schüttelkopf, A. W., \& Van Aalten, D. M. (2004). PRODRG: a tool for high-throughput crystallography of protein-ligand complexes. Acta Crystallographica Section D: Biological Crystallography, 60(8), 1355-1363. doi.org/10.1107/S090744490401167

Trott, O., \& Olson, A. J. (2010). AutoDock Vina: improving the speed and accuracy of docking with a new scoring function, efficient optimization, and multithreading. Journal of computational chemistry, 31(2), 455-461. doi.org/10.1002/jcc.21334 
Trede, N. S., Langenau, D. M., Traver, D., Look, A. T., \& Zon, L. I. (2004). The use of zebrafish to understand immunity. Immunity, 20(4), 367-379. doi.org/ 10.1016/S1074-7613(04)00084-6

Trede, N. S., Zapata, A., \& Zon, L. I. (2001). Fishing for lymphoid genes. Trends in immunology, 22(6), 302-307. doi.org/ 10.1016/S1471-4906(01)01939-1

Wallner, B., \& Elofsson, A. (2003). Can correct protein models be identified?. Protein science, 12(5), 1073-1086. doi.org/10.1110/ps.0236803

Wang, Z., Zhang, Q., Meng, F., Li, S., Xu, Q., \& Qi, Z. (2018). Characterization of the ligand binding of PGRP-L in half-smooth tongue sole (Cynoglossus semilaevis) by molecular dynamics and free energy calculation. Electronic Journal of Biotechnology, 31, 93-99. doi.org/10.1016/j.ejbt.2017.10.010

Werner, T., Liu, G., Kang, D., Ekengren, S., Steiner, H., \& Hultmark, D. (2000). A family of peptidoglycan recognition proteins in the fruit fly Drosophila melanogaster. Proceedings of the National Academy of Sciences, 97(25), 13772-13777. doi.org/10.1073/ pnas.97.25. 13772

Wiederstein, M. and Sippl, M.J. (2007). ProSA-web: interactive web service for the recognition of errors in three-dimensional structures of proteins. Nucleic acids research, 35(suppl_2), W407-W410. doi.org/10.1093/nar/gkm290

Webb, B., \& Sali, A. (2014). Comparative protein structure modeling using MODELLER. Current protocols in bioinformatics, 47(1), 5-6. doi.org/10.1002/0471250953.bi0506s47

Yoshida, H., Kinoshita, K., \& Ashida, M. (1996). Purification of a peptidoglycan recognition protein from hemolymph of the silkworm, Bombyx mori. Journal of Biological Chemistry, 271(23), 13854-13860. doi.org/10.1074/jbc.271.23.13854

Yoder, J. A., Nielsen, M. E., Amemiya, C. T., \& Litman, G. W. (2002). Zebrafish as an immunological model system. Microbes and Infection, 4(14), 1469-1478. doi.org/ 10.1016/S1286-4579(02)00029-1 
Table 1 Model validation statistics of zPGRP2 using various model evaluation servers.

\begin{tabular}{|l|l|c|}
\hline \multicolumn{1}{|c|}{ Servers } & \multicolumn{1}{|c|}{ Parameters } & Validation scores \\
\hline Procheck & Most favored regions (\%) & 93.3 \\
& Additional allowed regions (\%) & 5.0 \\
& Generously allowed regions (\%) & 0.0 \\
& Disallowed regions (\%) & 1.7 \\
\hline Verify 3D & Averaged 3D-1D score >=0.2(\%) & 89.08 \\
\hline ERRAT & Overall quality (\%) & 95.652 \\
\hline ProSA & Z score & -5.01 \\
\hline ProQ & LG score & 3.068 \\
& Max Sub & 0.258 \\
\hline Prove & Z score mean & 0.528 \\
\hline
\end{tabular}

Table 2 Molecular docking results of PGN ligands with zPGRP2 receptor using AutoDock Vina

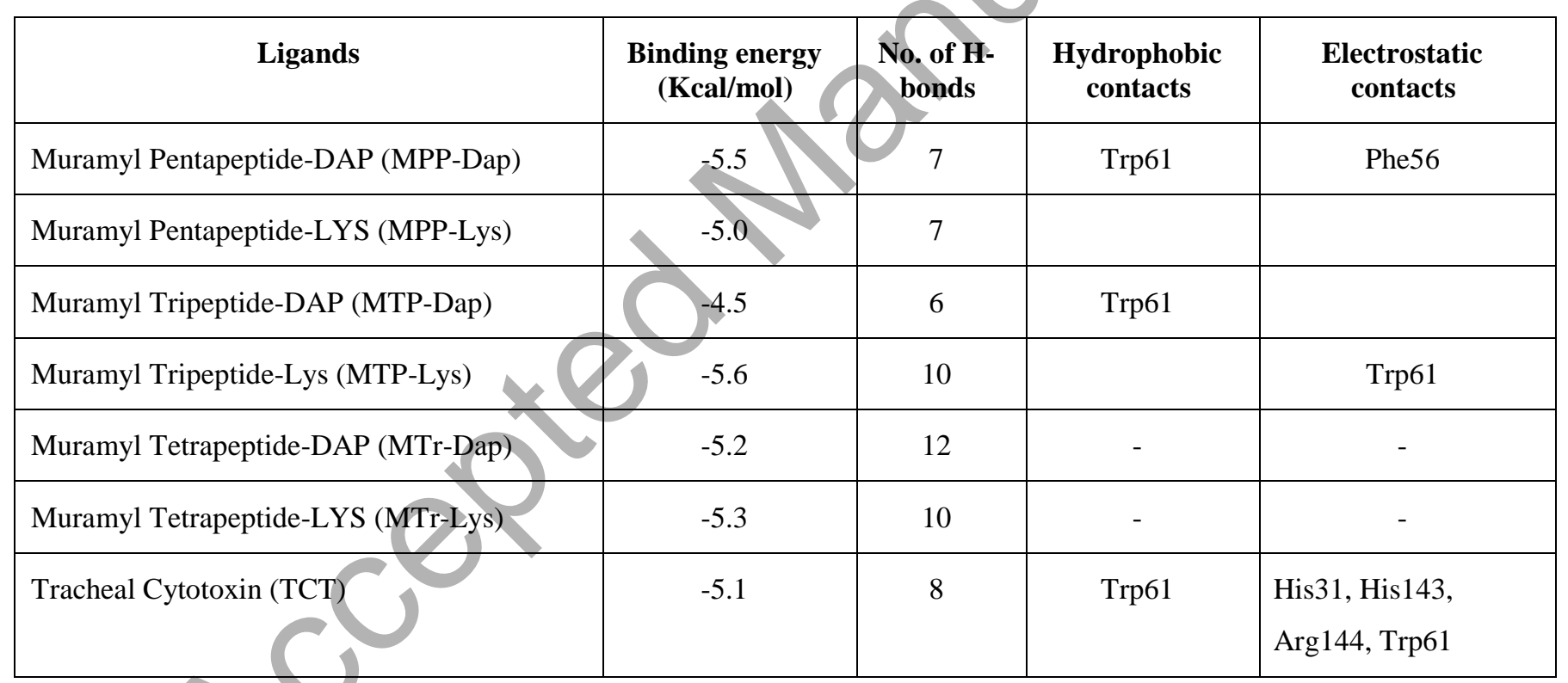


Table 3 Computed MM/PBSA binding free energies of zPGRP2-ligand complexes obtained from MD trajectories.

\begin{tabular}{|l|l|l|l|l|l|l|l|}
\hline BE Parameters & MPP-Dap & MPP-Lys & MTP-Dap & MTP-Lys & MTr-Dap & MTr-Lys & TCT \\
\hline $\begin{array}{l}\text { van der Waal } \\
\text { energy (kJ/mol) }\end{array}$ & $-222.595+/-$ & $-183.839+/-$ & $-184.231+/-$ & $-249.213+/-$ & $-207.199+/-$ & $-130.358+/-$ & $-184.807+/-$ \\
\hline $\begin{array}{l}\text { Electrostatic } \\
\text { energy (kJ/mol) }\end{array}$ & $-28.249+/-$ & $-81.490+/-$ & $-133.378+/-$ & $-129.732+/-$ & $-139.322+/-$ & $-30.380+/-$ & $-364.987+/-$ \\
\hline $\begin{array}{l}\text { Polar solvation } \\
\text { energy (kJ/mol) }\end{array}$ & $265.200+/-$ & $329.672+/-$ & $316.551+/-$ & $354.638+/-$ & $342.829+/-$ & $222.528+/-$ & $341.399+/-$ \\
\hline $\begin{array}{l}\text { SASA energy } \\
(\mathrm{kJ} / \mathrm{mol})\end{array}$ & $-20.585+/-$ & $-20.477+/-$ & $-18.844+/-$ & $-23.915+/-$ & $-19.725+/-$ & $-12.614+/-$ & $-19.908+/-$ \\
\hline $\begin{array}{l}\text { Binding energy } \\
(\mathrm{kJ} / \mathrm{mol})\end{array}$ & 0.131 & 0.299 & 0.142 & 0.114 & 0.202 & 0.230 & 0.928 \\
\hline
\end{tabular}



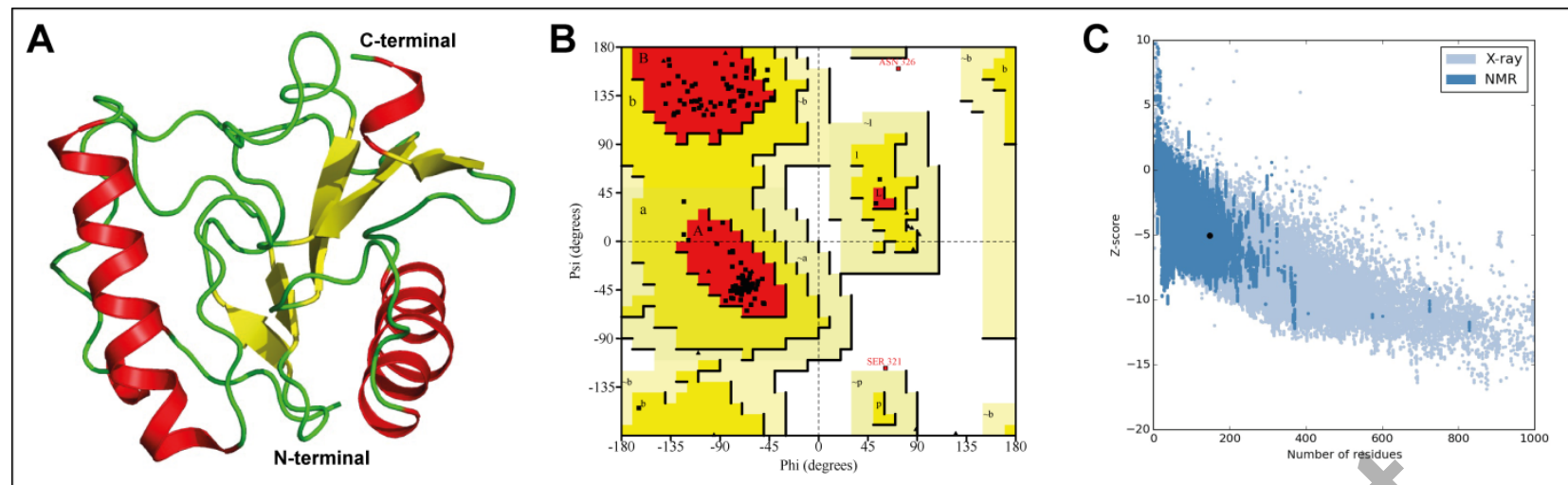

Figure 1 Homology modeling of PGRP2 from Zebrafish. (A) The solid ribbon cartoon representation of the 3-Dimensional structure of zPGRP2. (B) Ramachandran plot analysis of the modeled zPGRP2 inferred using Procheck tool in SAVES server. The dark red region indicates the most favored region, yellow for allowed, light yellow shows generously allowed region and white for disallowed region. The distribution of phi and psi angles determines torsion angles. (C) ProSA-web z-score analysis displaying the overall quality of the zPGRP2 model. 

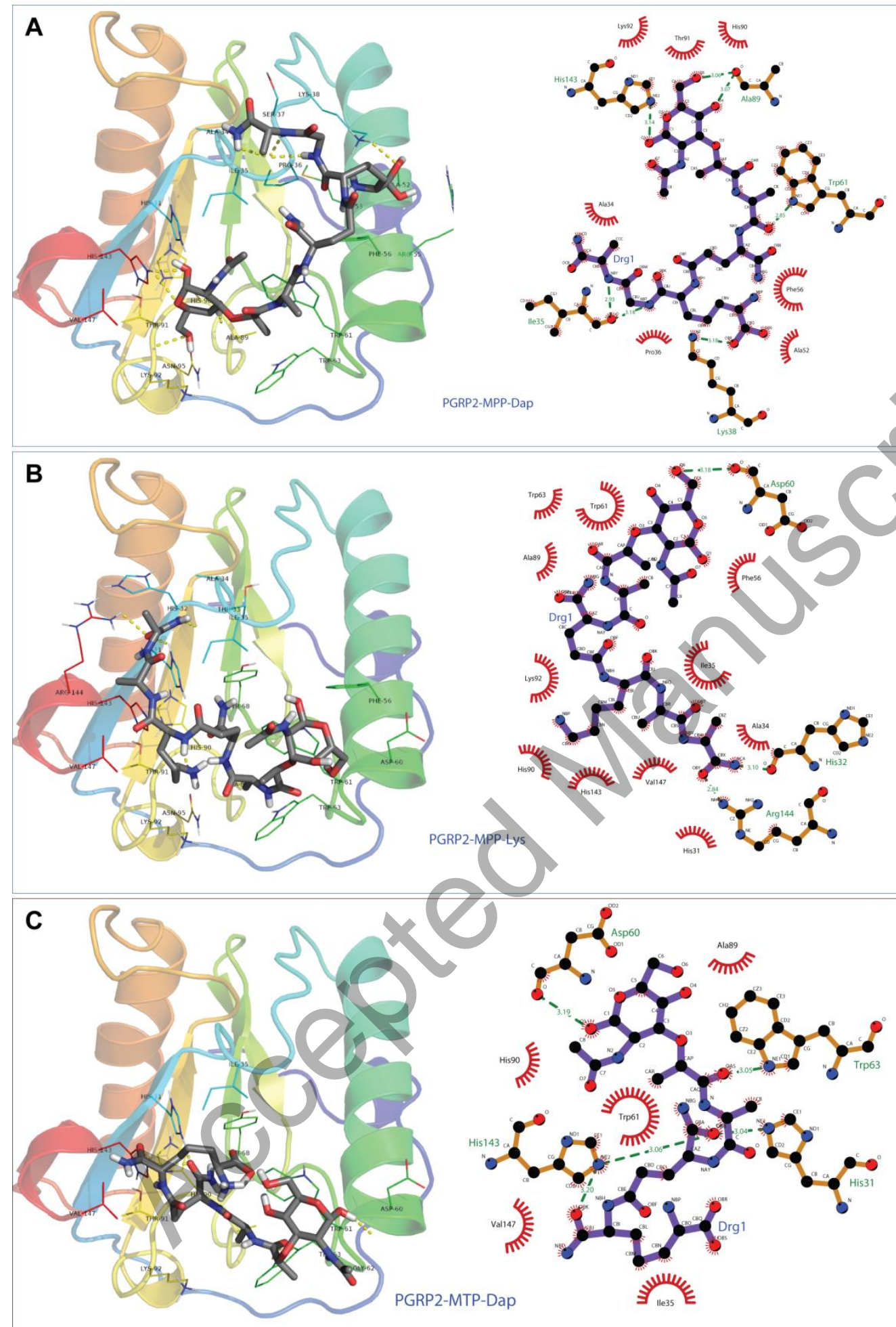

Figure 2 Molecular interaction analysis of (A) PGRP2- Muramyl Pentapeptide-DAP (MPP-Dap), (B) PGRP2- Muramyl Pentapeptide-LYS (MPP-Lys) and (C) PGRP2- Muramyl Tripeptide-DAP (MTP-Dap) using PyMOL and LigPlot+ software. 

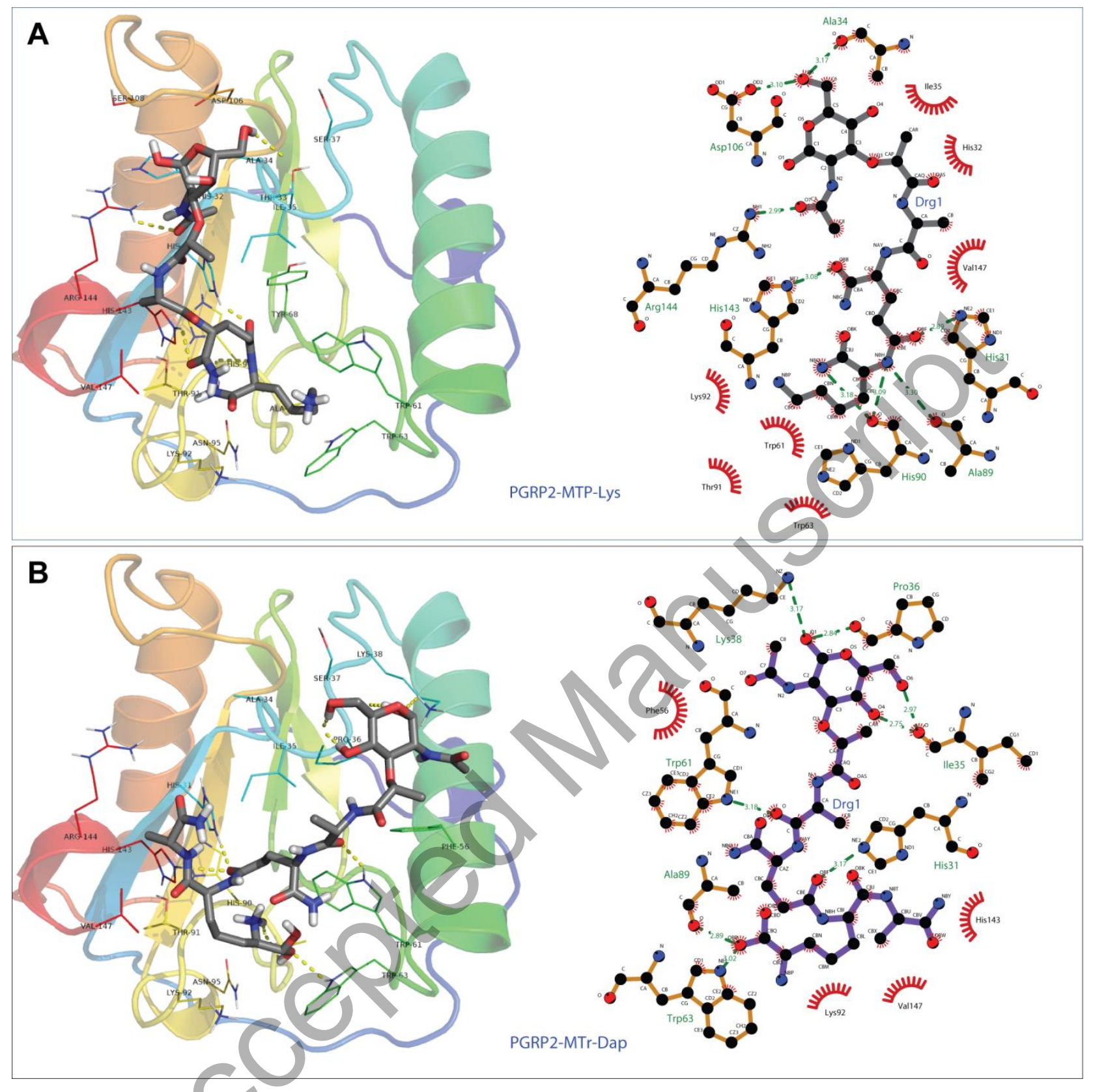

Figure 3 Molecular interaction analysis of (A) PGRP2- Muramyl Tripeptide-Lys (MTP-Lys) and (B) PGRP2- Muramyl Tetrapeptide-DAP (MTr-Dap). The hydrogen bonds are marked in dotted lines (yellow: left and green: right). 

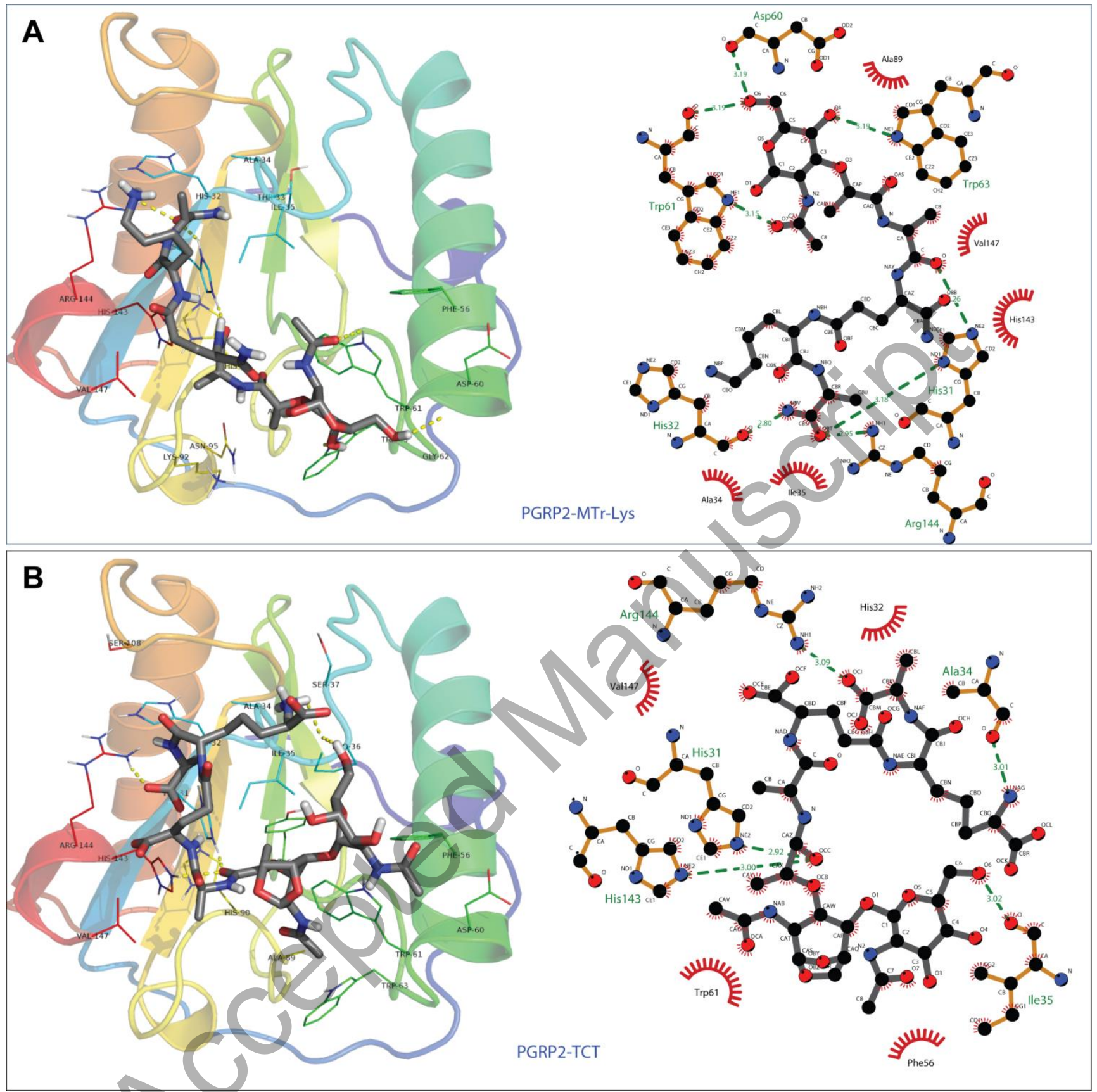

Figure 4 Molecular interaction analysis of (A) PGRP2-Muramyl Tetrapeptide-LYS (MTr-Lys) and (B) PGRP2-Tracheal Cytotoxin (TCT).The images were drawn using PyMOL and LigPlot ${ }^{+}$tools. 


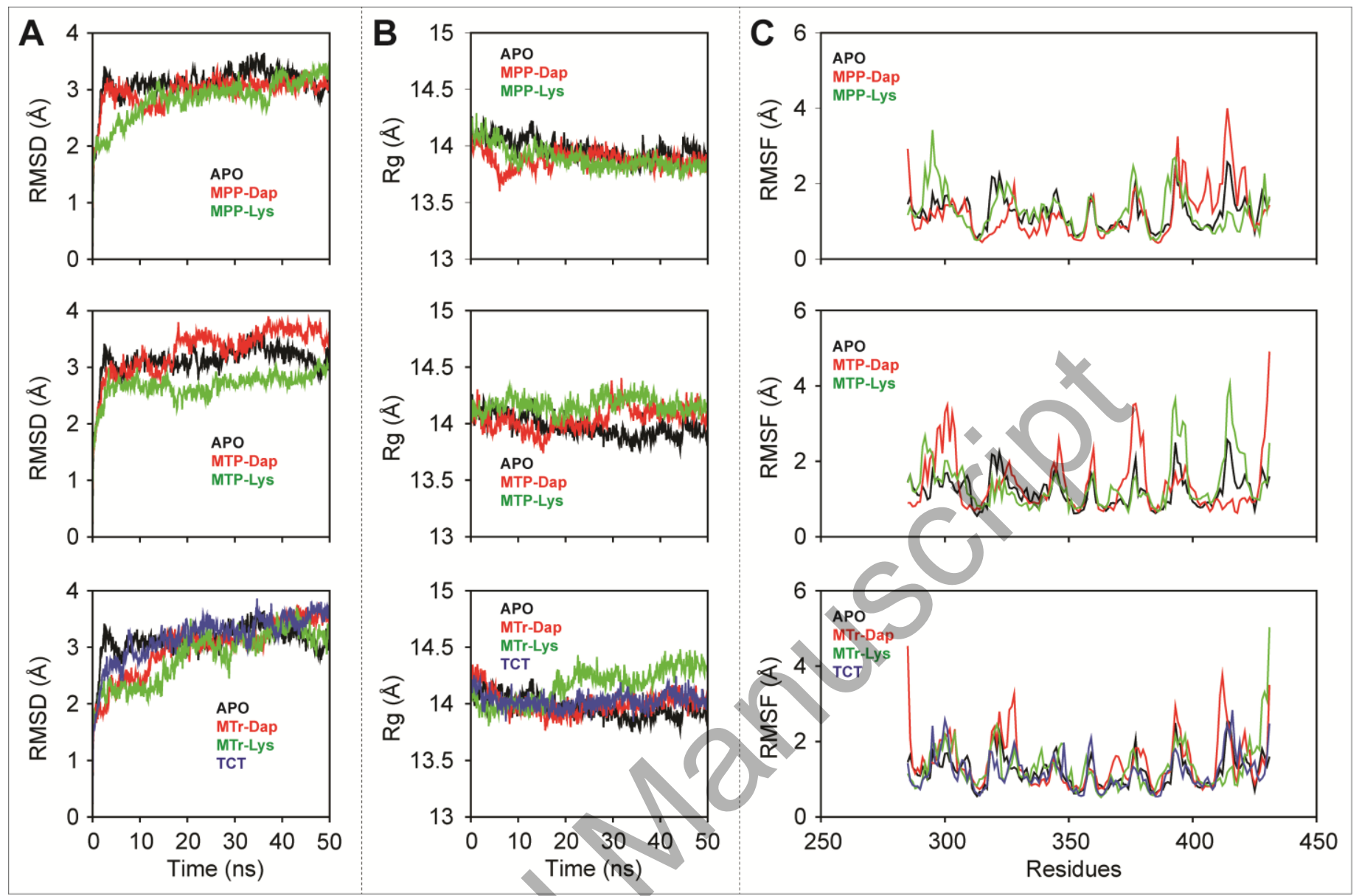

Figure 5 Stability parameters for Zebrafish PGRP2 homology model during 50ns MD simulation in Gromacs 5.1. (A) RMSD of C- $\alpha$ atoms, (B) Radius of gyration of protein, (C) RMSF of C- $\alpha$ atoms. 

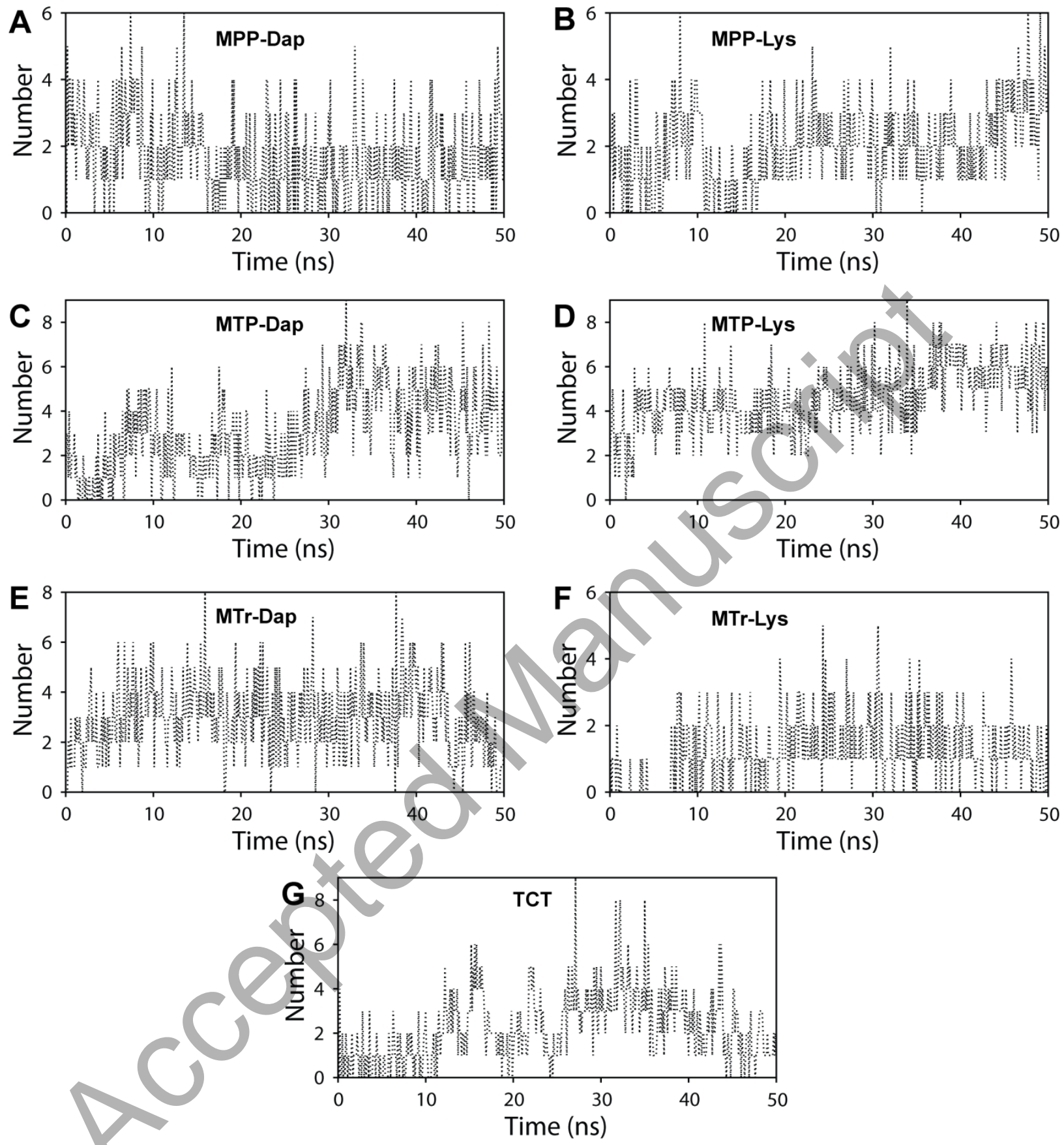

Figure 6 The dynamics of inter-molecular Hydrogen bond analysis in different zPGRP2-PGN ligand systems over the time scale of 50 ns MD, (A) MPP-Dap complexes, (B) MPp-Lys Complex, (C) MTP-Dap Complex, (D) MTP-Lys Complex, (E) MTr-Dap Complex, (F) MTr-Lys Complex, (G) Tracheal Cytotoxin (TCT). 


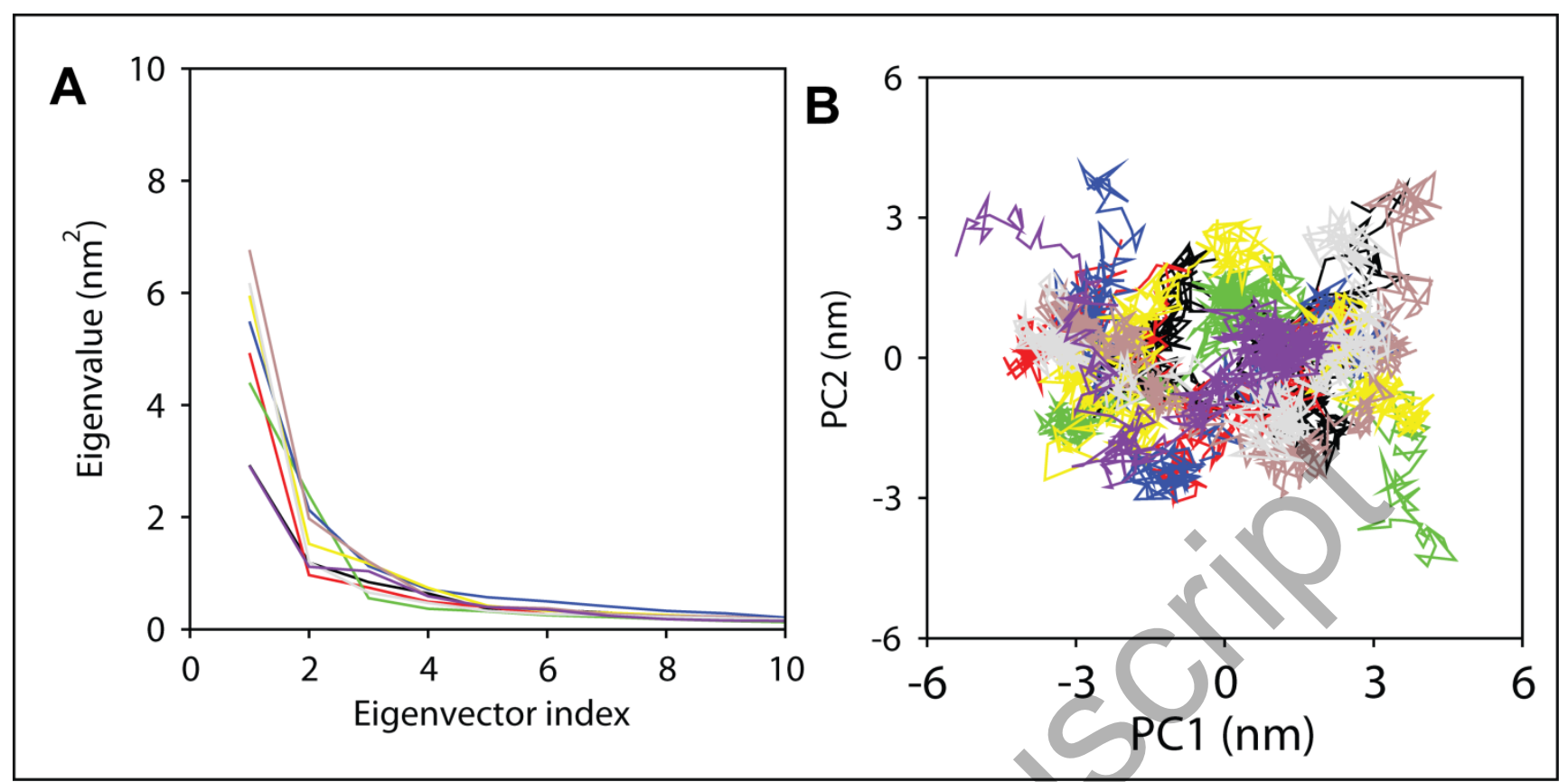

Figure 7 Principal component analysis of apo and holo conformers of zPGRP2 model systems using $50 \mathrm{~ns}$ trajectories of each system. PCA was conducted using g_covar and g_anaeig utility toolkits of GROMACS. (A) The eigenvalues plotted against the corresponding eigenvector indices obtained from the $\mathrm{C} \alpha$ covariance matrix constructed from the $50 \mathrm{~ns}$ MD trajectory (B) Projection of the motion of the apo and holo forms of PGRP2 in phase space along the first two principal eigenvectors (EV1 and EV2). 

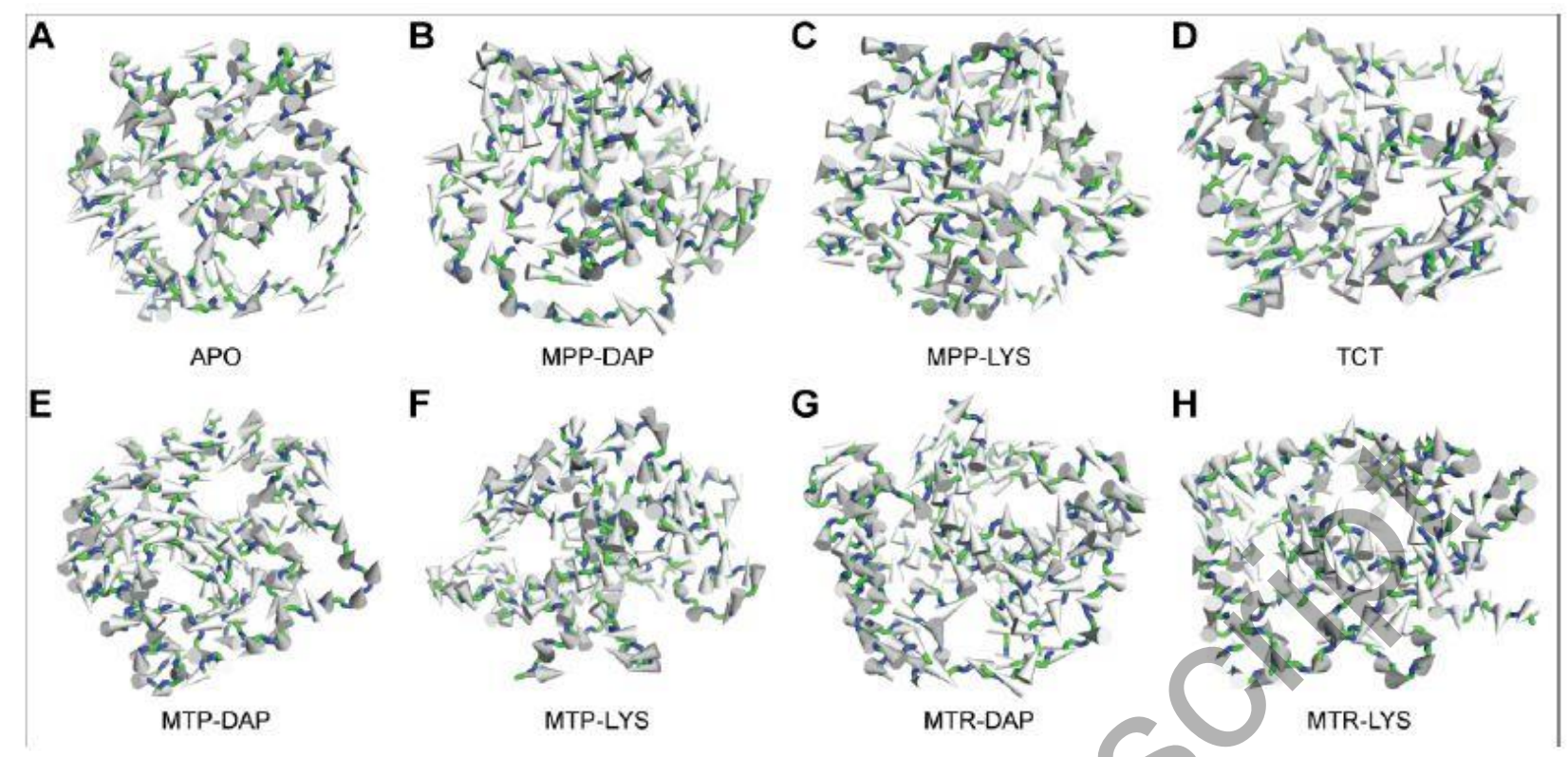

Figure 8 The Porcupine plot of the first eigenvector generated through principal component analysis of the representative structures of (A) APO (B) MPP-Dap complexes (C) MPp-Lys Complex (D) TCT Complex (E) MTP-Dap Complex (F) MTP-Lys (G) MTr-Dap Complex (H) MTr-Lys Complex The vectors represented as arrows, show the tendency of movement. 